\title{
Particulate and dissolved lipid classes in cultures of Phaeodactylum tricornutum grown in cage culture turbidostats with a range of nitrogen supply rates
}

\author{
Christopher C. Parrish \& Peter J. Wangersky \\ Department of Oceanography, Dalhousie University, Halifax, Nova Scotia B3H 4J1, Canada
}

\begin{abstract}
Production of intracellular and extracellular algal lipid classes was examined over a range of rates of supply of inorganic nitrogen. The marine diatom Phaeodactylum tricornutum was studied using 2 new techniques. Cage culture turbidostats wirc used for growing the phytoplankton. These are continuous culturing systems which provide a highly controlled algal environment. The Chromarod-Iatroscan TLC/FID system was used for lipid class separation and quantification. This permitted the resolution and detection of as many as 10 algal lipid classes plus an internal standard for accurate quantification. Up to 3 culturing units were supplied with medium of known nutrient content simultaneously, and particulate intracellular lipids together with dissolved lipids in the effluent media were monitored. Intracellular synthesis of a storage class, triglyceride, was clearly triggered by nitrogen stress, while synthesis of membrane-associated polar lipid classes was reduced under these conditions. Particulate triglyceride yield was higher at lower levels of nitrogen supply despite lower growth rates. Different amounts and different types of dissolved extracellular lipid classes were produced under nitrogen-stressed and nitrogen-replete conditions.
\end{abstract}

\section{INTRODUCTION}

Algal culture studies involving lipids are of interest in themselves, as well as in terms of projecting the results to aquatic ecosystems. An examination of factors affecting lipid production by microalgae in the laboratory should give insights into the production of compounds with high calorific value in the oceans. This information could also be used to manipulate the lipid content of algae grown in large-scale cultures to provide optimal lipid production for commercial applications.

This study is a new approach to the examination of lipid production by cultured algae. A new culturing unit has been used: the cage culture turbidostat. This system was recently introduced as a device for rapid determination of algal growth rate (Skipnes et al. 1980). In the present study a complete range of lipid classes has been measured in both particulate and dissolved fractions from turbidostats. Most previous studies have determined only the total lipid content of the phytoplankton or the fatty acids of some of the particulate lipid classes. A new analytical technique has been used to perform the lipid class measurements: the Chromarod-Iatroscan (TLC/FID) system which has recently seen considerable use in biomedical research (Ackman 1981). Parrish (1986) has given a detailed description of the analytical technique used here and its relationship to other techniques. Fig. 1 shows the relationship between the cage culture turbidostat and more commonly used culturing devices.

The advantages and disadvantages of the various culturing techniques shown in Fig. 1 are discussed by Parrish (1986). The principal advantage of the cage culture turbidostat is that the size of the phytoplankton population in the culture vessel can be kept constant under both nutrient-replete and nutrient-stressed conditions. The principal disadvantages are that the culture is hard to keep axenic and that the culturing system itself is relatively complicated.

The supply of nutrients plays a key role in algal growth in cultures and in the natural environment. In most aquatic environments the supply of nitrogen, phosphorus, or silicon is at least periodically growth- 
a

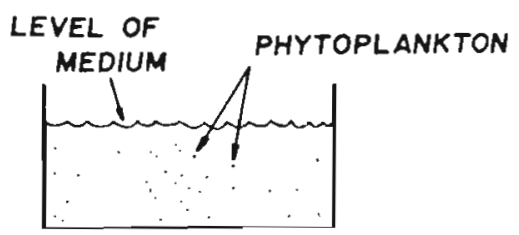

$b$

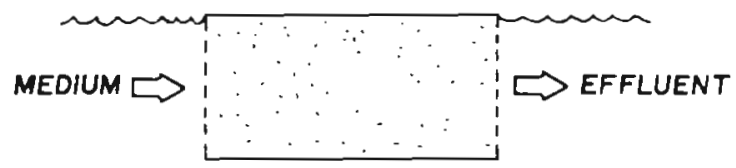

C
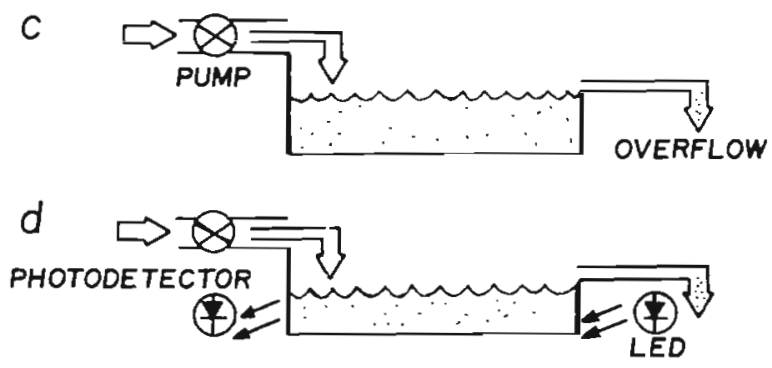

e

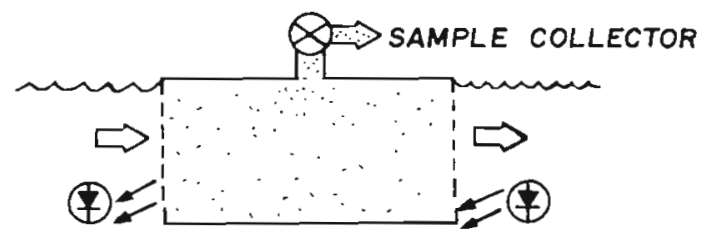

Fig. 1. Schematic representation of phytoplankton culturing systems. (a) Batch culture. (b) Dialysis or cage culture. (c) Chemostat: the medium is supplied at a constant rate. (d) Turbidostat: the medium is supplied to the culture by a pump controlled by a photodetector. (e) Cage culture turbidostat. the medium is supplied at a constant rate. Medium is removed from inside the culture by a pump controlled by a photodetector

limiting. There are several species of marine and freshwater phytoplankton which accumulate total lipid as a result of nitrogen or silicon starvation (Collyer \& Fogg 1955, Shifrin \& Chisholm 1981). However, an examination of data presented by these authors, and by Richardson et al. (1969), indicates that members of the same genus respond to nitrogen starvation differently, and that even members of the same species may not accumulate lipid as a result of nitrogen starvation in one type of culture system, while accumulating substantial amounts in another type. It seems likely that a factor other than simply a lack of nitrogen may be involved. It is also likely that since lipids are a heterogeneous group of compounds of varying structures and functions, the measurement of total lipids may be masking different trends occurring among the individual lipid classes.

There have been few studies on the effects of nutrients on lipid classes within phytoplankton cells. There have been even fewer on lipids released extracellularly by living cells (Hellebust 1974). Nutrient stress is believed to be one of the more important factors influencing extracellular release of organic compounds (Wangersky 1978, Jensen 1984). In the present study a complete range of intracellular and extracellular lipid classes has been measured in both nitrogen-stressed and nitrogen-replete cultures.

The alga used in this study was the marine diatom Phaeodactylum tricornutum. A survey by Ansell et al. (1964) of the first publications containing lipid data for this alga indicated that the intracellular lipid content was very variable. Values from 2 to $34 \%$ of dry weight were published between 1930 and 1964. Ansell et al. (1964) suggested that the available nitrogen supply was the most influential single factor causing this variation'. Recently, Thomas et al. (1984) found that although they could increase the lipid contant of $P$. tricornutum to $30 \%$ of dry weight under extreme nitrogen deficiency, there was no increase in the lipid yield. The present work with cage culture turbidostats examines the yield under a range of nitrogen supply rates.

\section{METHODS}

Three cage culture turbidostats (Manna Marine Enterprises, Halifax, Nova Scotia, Canada) were used to grow Phaeodactylum tricornutum with varying supply rates of nitrogen. Fig. $1 e$ is a diagrammatic representation of such a unit. These units are similar to those described by Skipnes et al. (1980) except in the location of the filters used to form the 2 ends of the cage and in the method of reversing flow across these filters to reduce clogging. The filters were held above the culture chamber to facilitate filter changes and a valve was placed in the medium delivery line so that flow through the chamber could be reversed while keeping a unidirectional flow from the medium reservoir as well as to the effluent receptacle.

The volume of the culture vessels was $400 \mathrm{ml}$ and approximately $3 \mathrm{l}$ of medium passed through each of the vessels per day. The medium supplied to the phytoplankton was a modified f/2 (Guillard \& Ryther 1962). The medium base consisted of Dalhousie Aquatron seawater which was dialysed for further purification. $\mathrm{NH}_{4} \mathrm{Cl}$ was used as the nitrogen source and the amount entering and leaving the turbidostats was monitored using a regularly calibrated ammonia electrode (Orion Research, Cambridge, Massachusetts, USA). The precision of these measurements was about $1 \% \mathrm{CV}$.

Cell concentrations were measured daily with a Coulter Counter (Model $1015 \mathrm{Z}_{\mathrm{B}}$ ). With the cell measurements and the nitrogen measurements it is possible to calculate the amounts of nitrogen offered to and taken up by each cell each day. The turbidostat 
cultures were held at a variety of nitrogen supply rates up to $62 \mathrm{pg}$ cell ${ }^{-1} \mathrm{~d}^{-1}$. The different supply-rate values were evenly spaced for most of the experiment, but were more closely spaced at the lower end of the scale: cultures were held at 5 levels between 0.2 and $0.5 \mathrm{pg}$ cell ${ }^{-1} \mathrm{~d}^{-1}$.

The threshold at which the turbidity-controlled pump harvested cells from the medium was maintained at the same level for all 3 culture vessels throughout the experiment. Growth rate, $\mu$, was calculated as the proportion of the turbidostat volume harvested in a day. Culture temperature was held at $20^{\circ} \mathrm{C}$, and light intensity at $70 \mu \mathrm{E} \mathrm{m} \mathrm{m}^{-2} \mathrm{~s}^{-1}$ with a light-dark cycle of $16: 8 \mathrm{~h}$. These conditions were chosen because they resulted in the smallest daily variability in harvest volumes.

The product of the specific growth rate and the lipid class composition of the cells was used to estimate particulate yields. Yields of dissolved lipid classes were obtained by measuring the lipid class concentration in the incoming medium and in the effluent media from the turbidostats. Lipid class analyses were performed on dichloromethane extracts on an Iatroscan MK III (Iatron Laboratories, Tokyo) using modifications of previously described procedures (Delmas et al. 1984, Parrish \& Ackman 1985). Table 1 shows the range of classes that can be measured with this TLC/FID technique.

Table 1. Phaeodactylum tricornutum. Lipid class composition ( $\pm 95 \%$ confidence intervals, $n=10$ ) of nitrogen-replete cells, determined by Chromarad thin-layer chromatography (TLC) with Iatroscan flame ionization detection (FID). Abbreviations for classes given in parentheses

\begin{tabular}{|lc|}
\hline \multicolumn{1}{|c|}{ Lipid class } & Content (pg cell $\left.{ }^{-1}\right)$ \\
\hline Aliphatic hydrocarbons (HC) & $0.09( \pm 0.06)$ \\
Sterol esters and wax esters (WE) & $0.04( \pm 0.04)$ \\
Methyl esters and other short-chain & $0.02( \pm 0.01)$ \\
esters (ME) & $0.11( \pm 0.04)$ \\
Triglycerides (TG) & $0.21( \pm 0.08)$ \\
Free fatty acids (FFA) & $0.06( \pm 0.02)$ \\
Free aliphatic alcohols (ALC) & $0.06( \pm 0.02)$ \\
Free sterols (ST) & $0.03( \pm 0.03)$ \\
Diglycerides (DG) & $0.95( \pm 0.16)$ \\
Acetone-mobile polar lipids (AMPL) & $0.98( \pm 0.19)$ \\
Phospholipids and other acetone- & \\
immobile polar lipids (PL) & \\
- AMPL includes chlorophyll a (CHL) & \\
\hline
\end{tabular}

Dissolved lipids were extracted from between $250 \mathrm{ml}$ and 11 of seawater. Particulate samples were usually obtained by filtering $50 \mathrm{ml}$ of phytoplankton culture $\left(10^{6}\right.$ cells $\left.\mathrm{ml}^{-1}\right)$. These samples were extracted by grinding the glass fibre filter, initially in dichloro- methane-methanol $(2: 1)$, and subsequently in $100 \%$ dichloromethane. The extracts were washed with distilled water that had been passed through a Millipore Super- $Q$ system and then heated to $800^{\circ} \mathrm{C}$ to further purify it. Samples were spiked with an internal standard, hexadecan-3-one, immediately after filtration, and total procedure blanks were run on each day of analysis. Blank levels for most lipid classes were usually below the detection limit of the analytical procedure. Several samples were extracted without the addition of the internal standard to check that there were no significant levels of any compounds that could interfere chromatographically with hexadecan-3-one.

Yields of dissolved lipids were obtained for cultures at nitrogen supply rates around 0.4 and $30 \mathrm{pg} \mathrm{cell}^{-1} \mathrm{~d}^{-1}$. Particulate lipids were measured at the extremes of nitrogen-stressed and nitrogen-replete conditions and at several points in between. Chlorophyll a (CHL) was measured daily in a Turner fluorometer.

\section{RESULTS}

The amount of nitrogen offered to the cells, the amount used by each cell, and the surplus in the turbidostat effluent were all closely correlated (Table $2)$. The cells continued to take up more nitrogen per day as the supply increased, but at the same time a greater proportion of the nitrogen supplied was passing through the culture vessel unused. At low nitrogen supply rates the proportion of nitrogen in the incoming medium used by the cells was much higher than at high supply rates.

Most cultures used either 5 to $10 \%$ of the nitrogen supplied or else between 80 and $90 \%$; no cultures used between 18 and $48 \%$ of the nitrogen supplied. This polarization in the proportion of nitrogen used suggested a means of categorising the cells grown in this experiment. Cells using less than $18 \%$ have been assumed to be growing under nitrogen-replete conditions; those using more than $48 \%$ have been assumed to be nitrogen-stressed.

TLC/FID chromatograms for the 2 types of cells were different in one major respect: triglyceride was a minor constituent of cells grown under nitrogen-replete conditions, but a major constituent in nitrogen-stressed cells (Fig. 2). The pigment-containing AMPL peak (abbreviations are explained in Table 1) was significant in all chromatograms, as was the PL peak. HC, FFA, different types of alcohols and different types of esters were also detected in smaller amounts in most samples. Table 1 gives the mean levels of particulate classes detected in nitrogen-replete cultures.

Occasionally, Phaeodactylum tricornutum growing in nitrogen-deficient media would change from its 
Table 2. Phaeodactylum tricornutum. Pearson product moment linear correlation coefficients for the complete range of nutrient conditions offered. Abbreviations explained in Table 1

\begin{tabular}{|c|c|c|c|c|c|c|c|}
\hline & $N$ offered & $N$ used & $\% \mathrm{~N}$ used & Surplus $N$ & Growth & $\mathrm{CHL}$ & $\mathrm{TG}$ \\
\hline$N$ used $\left(p g c e l^{-1} d^{-1}\right)$ & 0.92 & & & & & & \\
\hline$\% \mathrm{~N}$ used & -0.74 & -0.68 & & & & & \\
\hline Surplus $N\left(\right.$ ug-at l ${ }^{\prime}$ ) & 0.97 & 0.84 & -0.80 & & & & \\
\hline Growth $\left(\mathrm{d}^{-1}\right)$ & 0.75 & 0.80 & -0.70 & 0.71 & & & \\
\hline CHL (pg cell ${ }^{-1}$ ) & 0.92 & 0.88 & -0.86 & 0.92 & 0.82 & & \\
\hline TG $\left(\mathrm{pgcel} \mathrm{l}^{-1}\right)$ & -0.65 & -0.66 & 0.89 & -0.67 & NS & -0.71 & \\
\hline ST (pg cell $\left.{ }^{-1}\right)$ & 0.63 & 0.73 & NS & NS & 0.73 & 0.68 & NS \\
\hline AMPL (pg cell ${ }^{-1}$ ) & 0.72 & 0.77 & -0.76 & 0.72 & 0.80 & 0.84 & -0.66 \\
\hline PL (pg cell ) & NS & NS & -0.79 & NS & NS & 0.61 & -0.71 \\
\hline Total lipids (pg cell ${ }^{-1}$ ) & NS & NS & -0.67 & NS & 0.73 & 0.68 & NS \\
\hline
\end{tabular}

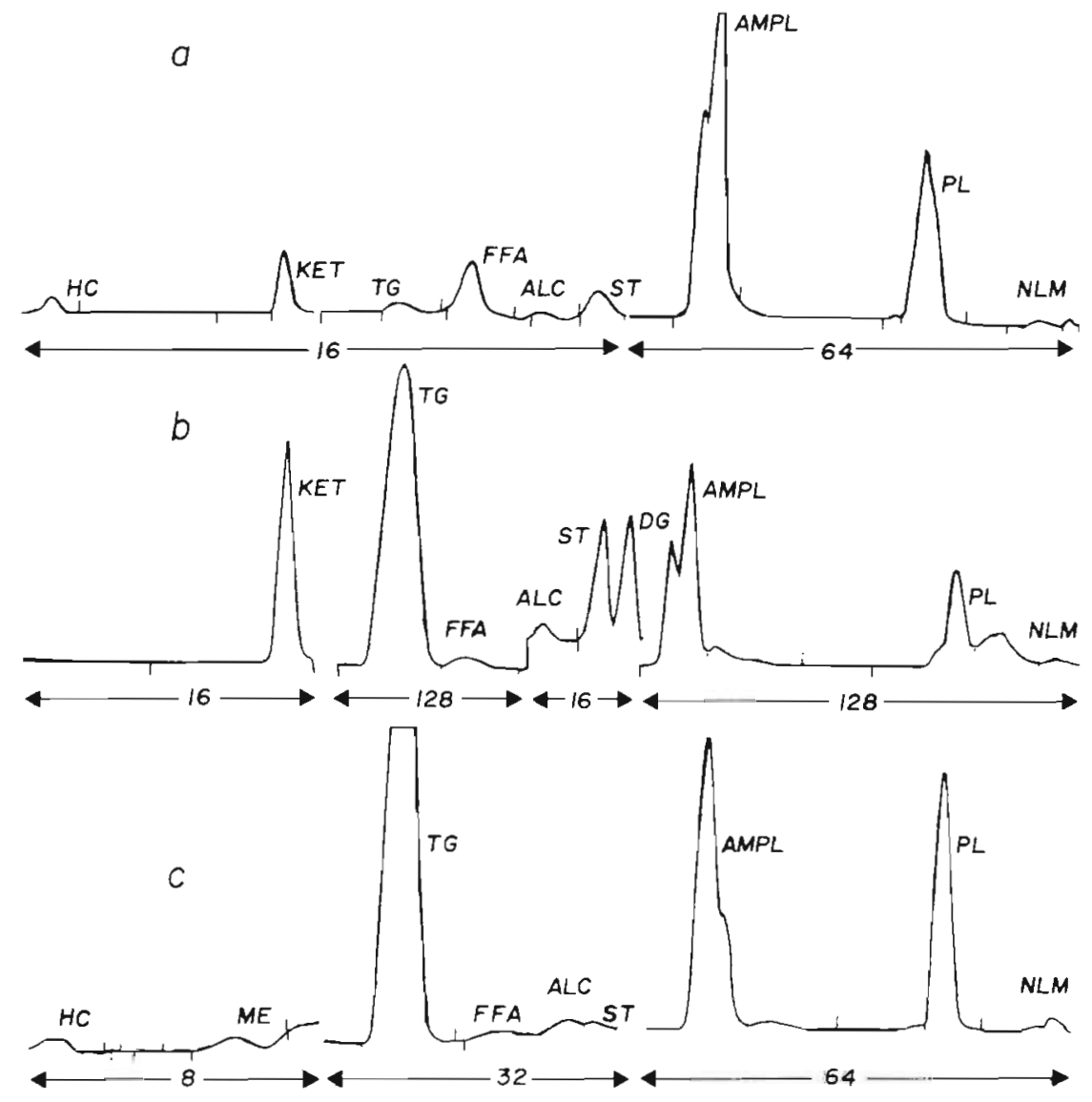

Fig. 2. Phaeodactylum tricornutum. TLC/ FID chromatograms of particulate lipids from cells grown in nitrogen-deficient and nitrogen-replete media. Abbreviations explained in Table 1. Recorder attenuation is given under each chromatogram in mV full-scale deflection. (a) Unicellular organisms grown under nitrogen-replete conditions, (b) Unicellular organisms grown under nitrogen-deficient conditions. (c) Chainform obtained from a nitrogen-deficient culture. No internal standard (KET) was added to this sample normal unicellular forms to a chain-form. The lipids in this chain-form were measured on 2 separate occasions. TLC/FID chromatograms for the chain-form were very similar to those obtained from nitrogendeficient unicellular P. tricornutum (Fig. 2b, c).

TLC/FID chromatograms of the dissolved lipid classes from nitrogen-deficient and nitrogen-replete cultures were quite similar, and not very different from those of the medium supplied to these cultures (Fig. 3). For several of the classes 1-way analysis of variance indicated no significant difference in lipid class concentration before and after passing through the turbidostats. Where there was a significant difference the production rate per cell has been calculated (Table 3). The variability in nitrogen supply per cell in Table 3 reflects small differences in cell numbers and flow 
Fig. 3. TLC/FID chromatograms of dissolved lipids in ingoing medium and effluents from cage cultures grown under nitrogen-deficient and nitrogen-replete conditions. Attenuation for all 3 final scans is $32 \mathrm{mV}$ full-scale deflection. The preceding scans are all at a lower attenuation (mostly $16 \mathrm{mV}$ ). (a) Dissolved lipid class composition of ingoing medium: dialysed seawater. (b) Dissolved lipid classes in the effluent from a nitrogenreplete culture. (c) Dissolved lipid classes in the effluent from a nitrogendeficient culture

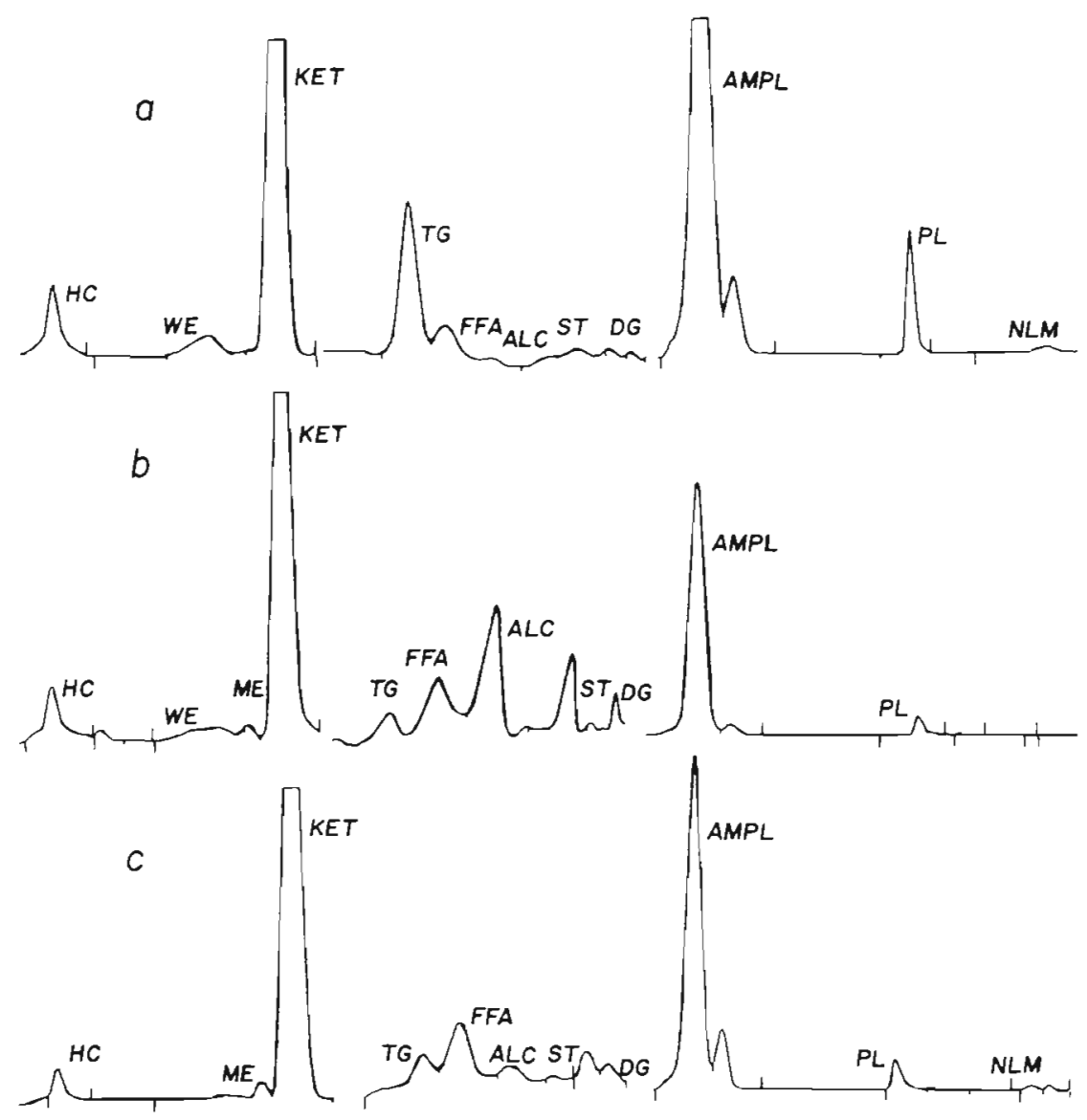

Table 3. Phaeodactylum tricornutum. Production rates of the major particulate and dissolved lipid classes per cell under nitrogen-deficient and nitrogen-replete conditions. All values in pg cell ${ }^{-1} \mathrm{~d}^{-1} \pm 1$ standard deviation of the mean of 3 turbidostat measurements

\begin{tabular}{|c|c|c|c|c|}
\hline \multirow[b]{2}{*}{ Lipid class } & \multicolumn{3}{|c|}{ Nitrogen supply } & $31.5( \pm 3.5)$ \\
\hline & Particulate & Dissolved & Particulate & Dissolved \\
\hline Triglycerides & $0.27 \pm 0.01$ & NS & $0.07 \pm 0.01$ & NS \\
\hline Free fatty acids & $0.03 \pm 0.01$ & $0.03 \pm 0.01$ & $0.16 \pm 0.05$ & NS \\
\hline Free alcohols & $0.01 \pm 0.004$ & $0.02 \pm 0.01$ & $0.04 \pm 0.02$ & $0.03 \pm 0.01$ \\
\hline Diglycerides & $0.01 \pm 0.005$ & NS & $0.02 \pm 0.001$ & $0.03 \pm 0.002$ \\
\hline AMPL & $0.15 \pm 0.03$ & $0.07 \pm 0.02$ & $0.52 \pm 0.12$ & $0.06 \pm 0.01$ \\
\hline Phospholipids & $0.07 \pm 0.05$ & $0.01 \pm 0.005$ & $0.45 \pm 0.008$ & NS \\
\hline Total lipids & $0.59 \pm 0.06$ & $0.15 \pm 0.01$ & $1.29 \pm 0.29$ & $0.14 \pm 0.01$ \\
\hline
\end{tabular}

rates among the turbidostats. Variability in the particulate lipid production rates for the 3 cultures being supplied with the same medium was usually less than $30 \%$. Dissolved lipid production rates were more variable. This may be simply an analytical problem: there was a significant input of most lipid classes to the turbidostats (Fig. 3a), so that the differences between input and output concentrations were sometimes quite smail by comparison with the total amounts measured. One way around this would be to slow down the flow of medium, so that the concentration of dissolved lipid classes in the effluent would be higher. Nonetheless, even with small differences between input and output, the $\mathrm{CV}$ for most dissolved lipid class production rates was still below $35 \%$.

The cell growth rate continued to increase over the 
entire range of rates of nitrogen offered and nitrogen used (Fig. 4a). The data do, however, seem. to fall into 2 groups with a change of slope close to $2 \mathrm{pg} \mathrm{N}$-used cell ${ }^{-1} \mathrm{~d}^{-1}$. The data distribution suggests a Michaelis-

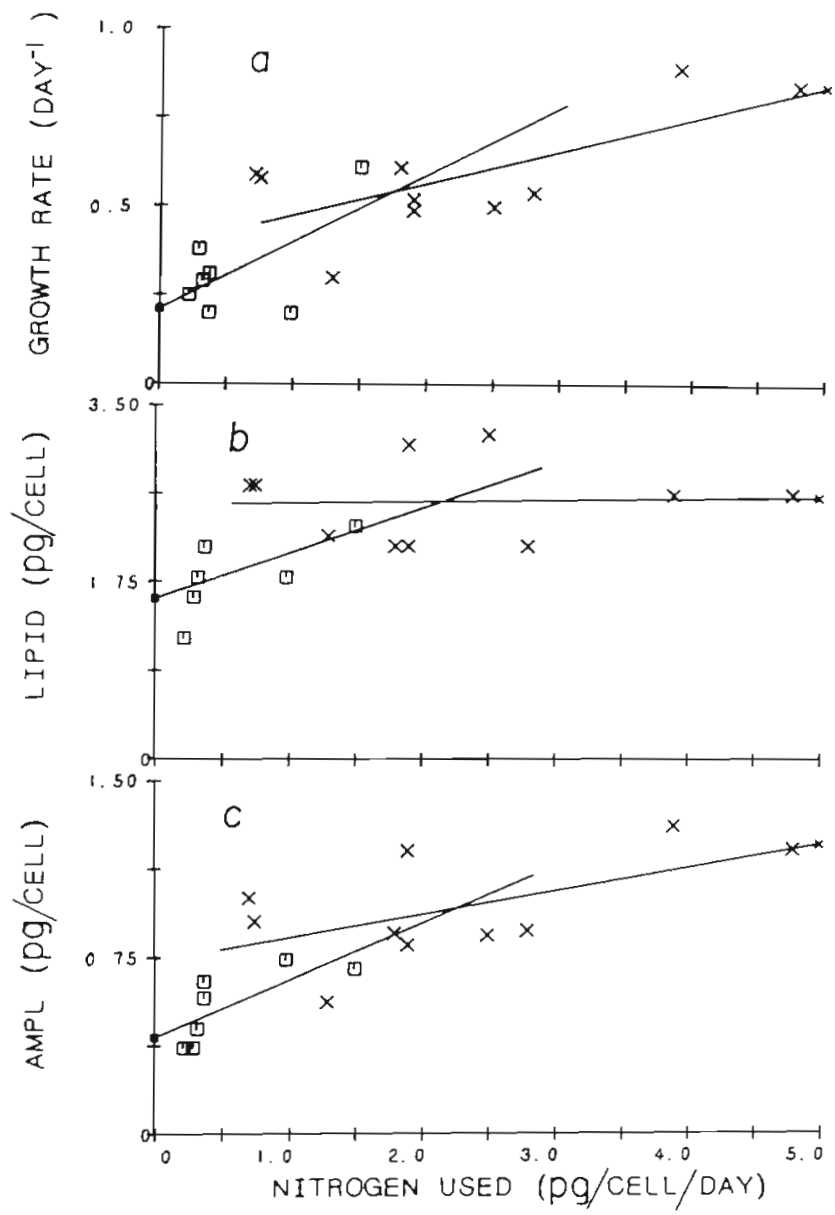

Fig. 4. Phaeodactylum tricornutum. Growth rate and lipid per cell as a function of rate of nitrogen use per cell. Crosses: nutrient-replete cells; squares: nutrient-deficient cells. (a) Growth rate, $\mu$, versus nitrogen use. (b) Sum of particulate lipid classes versus nitrogen use. (c) Acetone-mobile polar lipids versus nitrogen use

Menten curve would give a good fit; however, considerably more data would be needed to test this. For the purpose of this study, 2 linear regressions have been used to approximate the data distribution wherever there seemed to be a break in the slope. The choice of data points to include in the 2 regressions is based on the proportion of the nitrogen in the incoming medium used by the cells. Data from cells using more than $48 \%$ of incoming nitrogen have been grouped together, as have cells using less than $18 \%$ of incoming nitrogen.

In the case of growth rate versus nitrogen use, the correlation and slope of the nutrient-replete data points were both significant in this experiment $195 \%$ confidence level, $n=10$ ). Lipids per cell or per unit chlorophyll a were different in that their distributions tended to reach a plateau: none of the slopes or correlations of the nutrient-replete lipid data are significant in Fig. 4 \& 5. Thus, beyond an uptake rate of around 2 pg cell ${ }^{-1} \mathrm{~d}^{-1}$ the lipid composition appears to be inde-

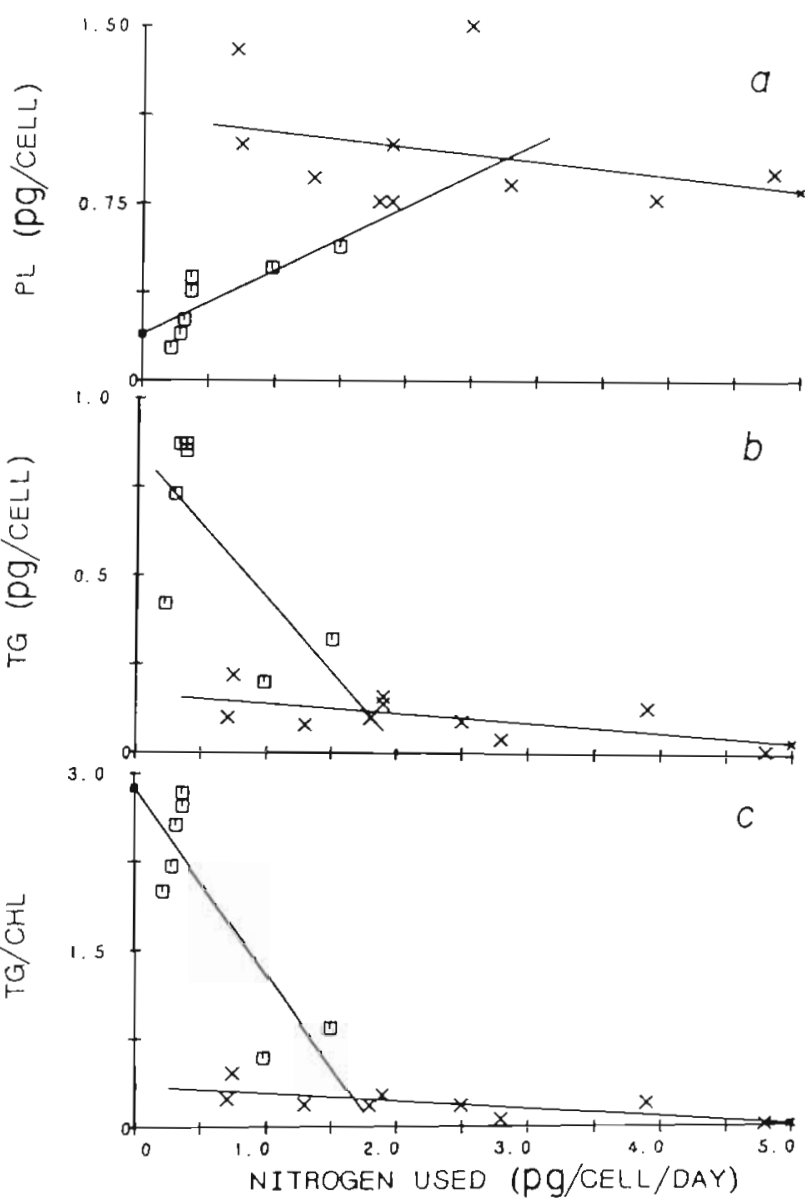

Fig. 5. Phaeodactylum tricornutum. Lipid classes as a function of the rate of nitrogen use per cell. Crosses: nutrient-replete cells; squares: nutrient-deficient cells. (a) Phospholipid per cell. (b) Triglyceride per cell. (c) Ratio of triglyceride to chlorophyll a

pendent of the rate of nitrogen use. The calculation of mean levels of lipids per cell in Table 1 is therefore justified.

Evidence of a plateau, or even a change in slope, was not however clearly apparent in all aspects of this data set (Fig. 6 to 8), although triglycerides per unit chlorophyll a did repeat the pattern of a significant negative slope followed by a levelling off (Fig. 7b).

Of all the classes measured, chlorophyll a, sterols, and AMPL were the only ones correlated with growth at the $99 \%$ confidence level (Table 2). This relation with growth seems to be quite linear (Fig. 8), and in the case of sterols the intercept is not significantly different from the origin. 


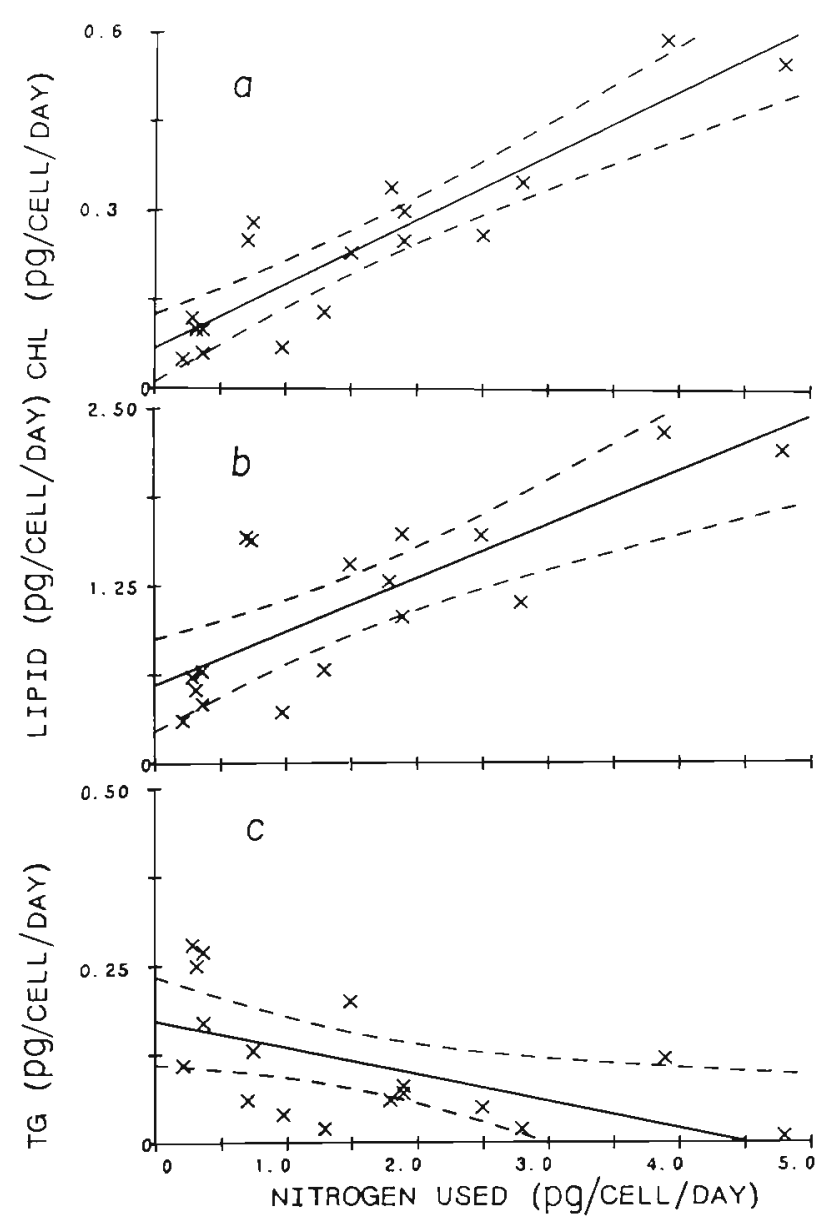

Fig. 6. Phaeodactylum tricornutum. Lipid production per cell as a function of nitrogen use per cell. Broken lines: $95 \%$ confidence intervals for regression lines. (a) Chlorophyll a production rate. (b) Sum of Iatroscan-measured particulate lipid classes. (c) Triglyceride production rate

\section{DISCUSSION}

The decrease in total lipids per cell and in total lipid productivity with increasing nitrogen stress (Fig. 4b \& 6b) appears to be in opposition to currently held views concerning lipid production in nitrogen-limited phytoplankton. The lack of any significant increase in the production of total dissolved lipids (Table 3 ) also does not seem to support currently held views concerning the production of dissolved organic matter by nitrogenstressed phytoplankton. What is clear from these data, however, is that there is a change in the types of lipids produced as Phaeodactylum tricornutum makes the transition from growing in nitrogen-replete to nitrogen-deficient media. Different classes of dissolved lipids are produced under these 2 conditions (Table 3 ). Particulate triglycerides per cell and triglycerides per unit chlorophyll a both increase significantly as the amount of nitrogen used per cell decreases (Fig. 5b, c).

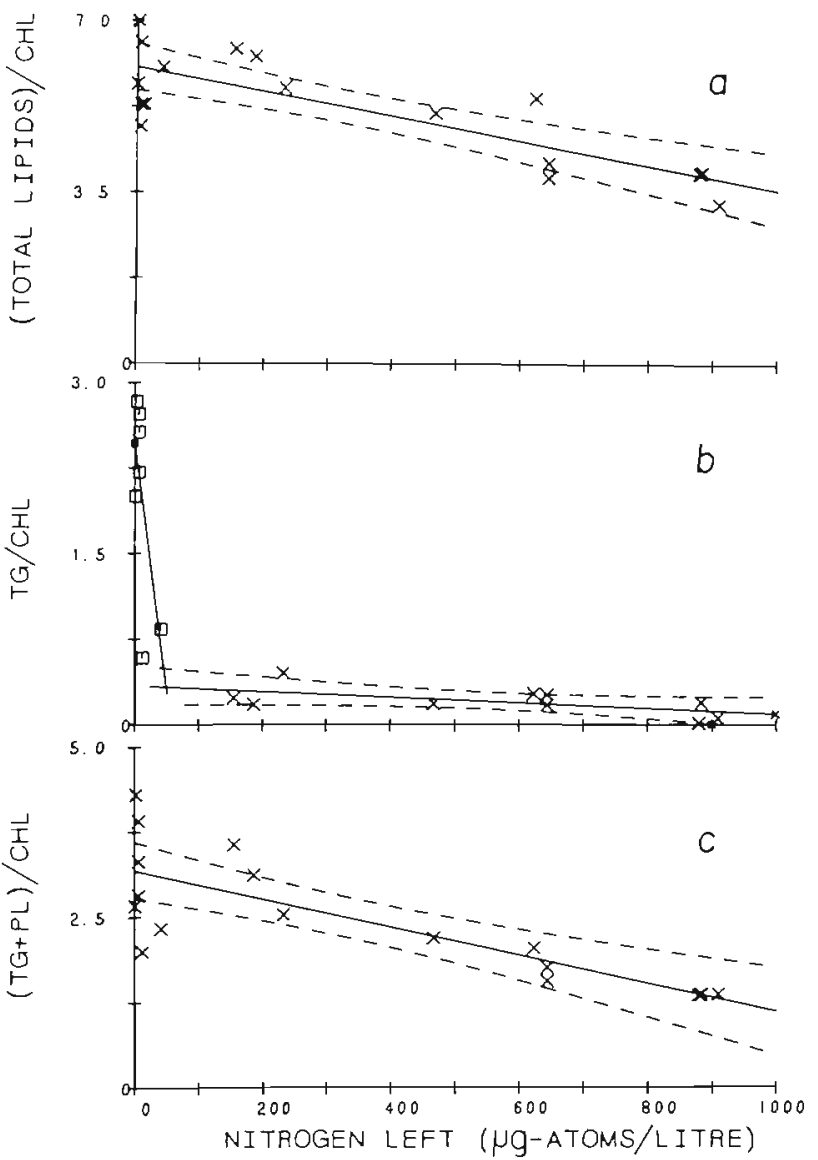

Fig. 7. Lipid per unit chlorophyll $a$ as a function of nitrogen concentration in effluent from turbidostats. Broken lines: $95 \%$ confidence intervals for regression lines. (a) Ratio of sum of Iatroscan-determined lipid classes to fluorometrically determined chlorophyll $a$. (b) Ratio of triglyceride to chlorophyll a. (c) Ratio of sum of triglyceride and phospholipid to chlorophyll $a$

By comparing total lipids to chlorophyll $a$ and plotting these data as a function of the concentration of nitrogen left in the medium (Fig. 7a), it is possible to make a direct comparison with more conventional measurements made in cultures and in the field. Chlorophyll $a$ is often used as a reference for measurements, if not numerically, at least conceptually. The course of phytoplankton blooms is usually followed by measuring chlorophyll a concentrations in the water column, and other organic classes are often expressed as a ratio to these concentrations. The concentration of surplus nitrogen in the turbidostat effluents most nearly approximates the concentration that would be measured in a sample of seawater taken from a culture or from the euphotic zone during a bloom. Under these conditions it can be seen that total lipids do indeed increase with increasing nitrogen stress (Fig. 7a). This is evidentiy the result of chlorophyll a levels in cells 


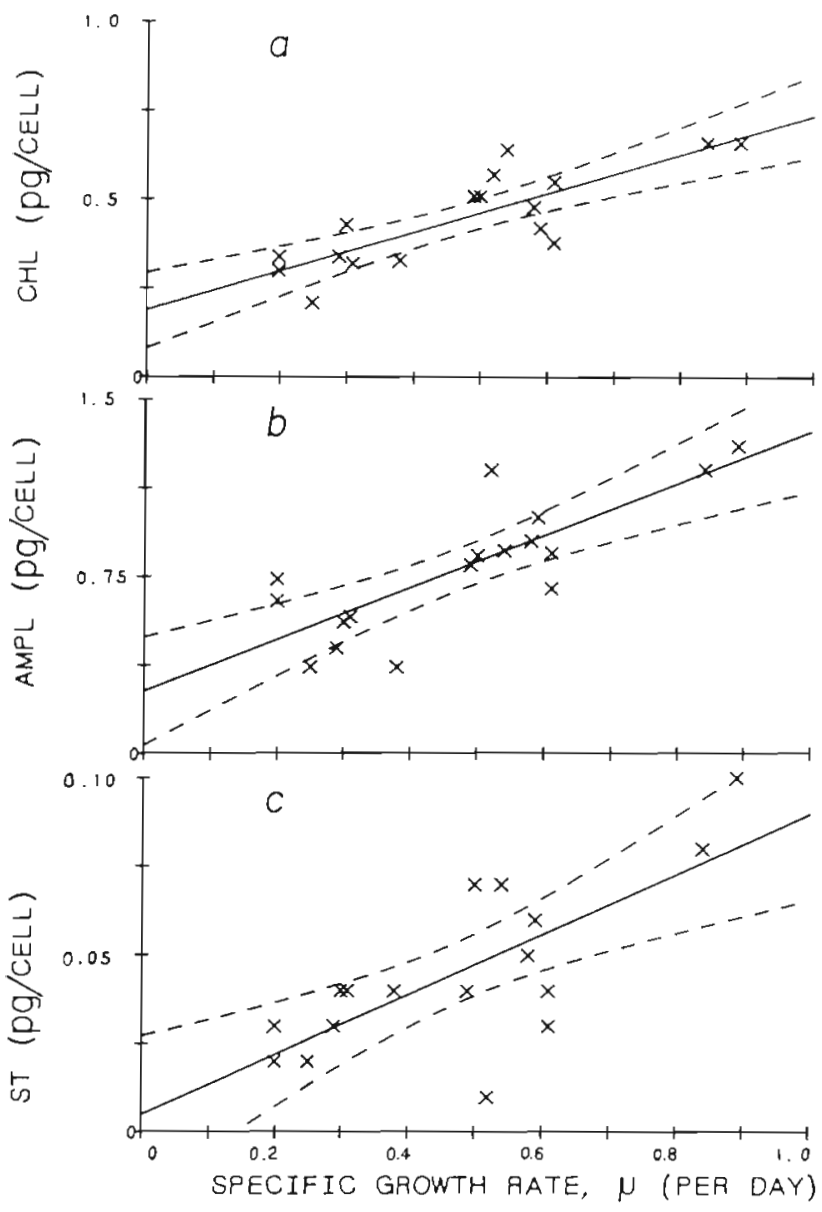

Fig. 8. Phaeodactylum tricornutum. Lipid classes as a function of growth rate. Broken lines: $95 \%$ confidence intervals for regression lines. (a) Chlorophyll a per cell. (b) Acetonemobile polar lipids per cell. (c) Sterols per cell

being more sensitive to nitrogen depletion than are the non-pigmented lipids.

The maximum level of total lipid was only 7 times the chlorophyll a concentration (Fig. 7 a). Since ratios of 2 orders of magnitude and more can be calculated from measurements made during diatom blooms in enclosed ecosystem bags (Morris et al. 1983) and in the field (Parrish 1987), expressing the present data in terms of ratios and nitrogen concentrations (Fig. 7a) does not seem to have resolved the dilemma. Nor is the answer simply that Phaeodactylum tricornutum is a species that does not respond dramatically to nitrogen deficiency. Similarly high lipid to chlorophyll a ratios can be calculated for $P$. tricornutum from data presented by Thomas et al. (1984). The answer probably lies in the culturing system used here. Richardson et al. (1969) were also unable to increase lipid content in their continuous cultures. Both Shifrin \& Chisholm (1981) and Thomas et al. (1984) employed combinations of batch and continuous cultures and found the highest Lipid content occurred after the longest period of time in nitrogen-free batch cultures.

In this cage culture study it was found that a supply rate of nitrogen any lower than the lowest used would result in the halting of cell division. Similarly, Richardson et al. (1969) found that they could not further reduce the nitrogen supply to their chemostats without losing the cultures. It would seem therefore, that phytoplankton cells have to stop dividing before there can be any massive accumulation of lipid. Microscopic observations by Opute (1974) suggest that diatom cells accumulate large levels of lipids at the end of the stationary phase in batch cultures. Opute (1974) also presented some evidence to suggest that an autotoxin may be responsible for the prevention of cell division at this stage. This would be consistent with the general observation that it is not possible to produce large accumulations of lipids in algae grown in continuous cultures, and that the greatest accumulation of lipids occurs when algae have been in the same medium for the greatest length of time.

\section{Intracellular lipid classes}

The strong negative correlation between triglycerides and phospholipids (Table 2) suggests a simple mechanism that would lead to the accumulation of triglycerides in nutrient-depleted cells. The major pathway of $\mathrm{CO}_{2}$ fixation in Phaeodactylum tricornutum is into 3-phosphoglycerate (Holdsworth \& Colbeck 1976). This is a direct precursor of phosphatidic acid, which is itself a precursor of both complex phospholipids and triglycerides (Gurr \& James 1980). Lecithins are the major phospholipids synthesized in $P$. tricornutum (Holdsworth \& Colbeck 1976). These phospholipids contain equimolar proportions of phosphorus and nitrogen, and so their synthesis in nitrogen-limited cells is likely to be hindered. In addition, the nitrogenous base cytidine is an essential cofactor in the biosynthesis of all complex phospholipids from phosphatidic acid (Gurr \& James 1980, Goodwin \& Mercer 1983). This importance of nitrogen in phospholipid biochemistry probably explains the decrease in phospholipid content in nitrogen-limited cultures (Fig. 5a). Since phospholipids and triglycerides have common precursors it is reasonable to assume that the common intermediates are likely to be channeled towards triglyceride synthesis if phospholipid synthesis is hindered.

The synthesis of triglyceride under conditions of low nitrogen supply and low nitrogen use is such a clear signal (Fig. 2, 5b, c, 6c \& $7 b$; Tables $2 \& 3$ ) that it may be possible to use triglyceride measurements to help indicate whether natural phytoplankton populations 
are stressed. High levels of triglycerides have indeed been found after the time of maximum measured chlorophyll a concentrations during spring blooms in Bedford Basin (Parrish 1987). Thus the determination of lipid class concentrations could provide a useful addition to the biochemical indices used to assess nitrogen deficiency in natural populations (Dortch et al. 1985).

The acetone-mobile polar lipids also gave a clear signal, but one that was different from that observed with triglycerides. The decrease in total lipid per cell with increasing nitrogen stress (Fig. 4b) reflects the pattern observed for AMPL (Fig. 4c) which was, on average, the major lipid component. This class of lipids accounted for $34( \pm 4) \%$ of lipids over the complete range of nitrogen supply rates. Chlorophyll a was clearly a component of this TLC band on the Chromarods, and by comparing fluorometric chlorophyll a values with AMPL values it would appear that CHL accounted for about $50 \%$ of the AMPL peak in nitrogen-replete cells. It is thus not surprising that these 2 values are correlated (Table 2), and that if one is correlated with growth so is the other (Fig. 8a, b). The strength of the relation with each other and with growth suggests, however, that the other $50 \%$ of the AMPL peak is also closely related to cell replication. The remaining components of this peak undoubtedly include glycolipids, the so-called 'chloroplast lipids' (Gurr \& James 1980). Specific types of glycolipids, the galactosyldiglycerides, are usually the predominant lipid in chloroplasts; they have been shown to be synthesized by the membranes of the chloroplast envelope (Goodwin \& Mercer 1983). These glycolipids have also been found to be the most important products of lipid synthesis in Phaeodactylum tricornutum (Holdsworth \& Colbeck 1976).

In 2 different cultures, Phaeodactylum tricornutum was observed to change from its normal unicellular forms to a chain form first reported by Coughlan (1962). It was unfortunately not possible to measure the amount of lipid per cell in this chain form, but the percentage composition was similar to that of the unicellular form taken from other cultures supplied with the same amount of nitrogen but at a different time during the experiment (Fig. 2). Thus, a chain form from a culture that had a slightly higher supply of nitrogen had less triglyceride and more polar lipid than the chain form from a more stressed culture. The chain forms occurred when the 2 turbidostats in operation at the time were being supplied with 2 different enrichments of nitrogen in dialysed seawater from the same source. It does not seem to be the exact amount of nitrogen that is critical in the formation of chains, nor were both these cultures below a threshold of nitrogen supply, since it was subsequently possible to maintain normal unicellular forms for extended periods at even lower nitrogen supply rates. It would seem that some other component in the base-medium was responsible for this transformation.

\section{Dissolved lipid production rates}

The absolute production rate of total dissolved lipid per cell does not seem to be influenced by nitrogen stress (Table 3). However, it does become a significantly larger proportion of the total particulate lipid produced. The proportion rose from about $10 \%$ to about $25 \%$ under nitrogen stress. These values are quite consistent with most estimates of the percentage of fixed carbon released extracellularly by natural populations of phytoplankton growing under nutrientreplete and nutrient-stressed conditions (Wangersky 1978). Increases in dissolved lipid concentrations after spring blooms (Kattner et al. 1983, Morris et al. 1983, Parrish 1987) could simply reflect the large numbers of cells present in the water columns immediately previously. Kattner et al. (1983) found large accumulations of polar lipids after blooms. In Bedford Basin, Nova Scotia, phospholipids were found to be a significant contributor to the increase in polar lipids after the bloom (Parrish 1987). Elevated levels of dissolved phospholipid production as a result of nitrogen limitation (Table 3) might seem incongruous. However, if nitrogen limitation hinders the production of complex particulate phospholipids from phosphatidic acid (which does not contain nitrogen), then a build-up of intracellular phosphatidic acid is likely to occur. Part of this could go to the production of triglycerides, the rest could presumably be released extracellularly.

The production of free fatty acids was also significantly higher in nitrogen-stressed cells (Table 3). This might be an artefact related to the significant increase in phospholipid production, since it is possible that a portion of the extracellular phospholipids is rapidly hydrolysed to produce free fatty acids.

\section{SUMMARY AND CONCLUSION}

In Phaeodactylum tricornutum synthesis of a storage component, triglycerides, is clearly triggered by nitrogen stress. The yield of triglycerides is higher at the lower levels of nitrogen supply and nitrogen use per cell despite lower growth rates.

The membrane-associated polar lipid classes are also affected by nitrogen stress. Their levels, however, are reduced. This results in the total lipid per cell decreasing with a decreasing supply of nitrogen to the cage cultures. The production rate of total dissolved 
lipids does not seem to be affected by stress; however, there are differences in the types of dissolved classes produced under nutrient-stressed and nutrient-replete conditions

Acknowledgements. Excellent technical assistance by R. L. Maass and the use of the Dalhousie Aquatron facility are gratefully acknowledged. This work was supported by grants from the Natural Sciences and Engineering Research Council to PJW. An Izaak Walton Killam Memorial Scholarship supported CCP.

\section{LITERATURE CITED}

Ackman, R. G. (1981). Flame ionization detection applied to thin-layer chromatography on coated quartz rods. In: Lowenstein, J. M. (ed.) Methods in enzymology, Vol. 72. Academic Press, London, p. 205-252

Ansell, A. D., Coughlan, J., Lander, K. F., Loosmore, F. A. (1964). Studies on the mass culture of Phaeodactylum. IV. Production and nutrient utilization in outdoor mass culture. Limnol. Oceanogr. 9: 334-342

Collyer, D. M., Fogg. G. E. (1955). Studies on fat accumulation by algae. J. exp. Bot. 6: 256-275

Coughlan, J. (1962). Chain formation by Phaeodactylum. Nature, Lond. 195: 831-832

Delmas, R. P., Parrish, C. C., Ackman, R. G. (1984). Determination of lipid class concentrations in seawater by thinlayer chromatography with flame ionization detection Analyt. Chem. 56: 1272-1277

Dortch, Q., Clayton, J. R., Thoresen, S. S., Cleveland, J. S., Bressler, S. L., Ahmed, S. I. (1985). Nitrogen storage and use of biochemical indices to assess nitrogen deficiency and growth rate in natural plankton populations. J. mar. Res. 43: 437-464

Goodwin, T. W., Mercer, E. I. (1983). Introduction to plant biochemistry. Pergamon, Oxford

Guillard, R. R. L., Ryther, J. H. (1962). Studies of marine planktonic diatoms I. Cyclotella nana Hustedt, and Detonula confervacea (Cleve) Gran. Can. J. Microbiol. 8: 229-239

Gurr, M. I., James, A. T. (1980). Lipid biochemistry: an introduction. 3rd edn. Chapman and Hall, London

Hellebust, J. A. (1974). Extracellular products. In: Stewart,
W. D. (ed.) Algal physiology and biochemistry. Univ. of California, Berkeley and Los Angeles, p. 838-836

Holdsworth, E. S., Colbeck, J. (1976). The pattern of carbon fixation in the marine unicellular alga Phaeodactylum tricornutum. Mar. Biol. 38: 189-199

Jensen, A. (1984). Excretion of organic carbon as a function of nutrient stress. In: Holm-Hansen, O., Bolis, L., Gilles, R. (ed.) Marine phytoplankton and productivity. SpringerVerlag, Berlin, p. 61-72

Kattner, G., Gercken, G., Hammer, K. D. (1983). Development of lipids during a spring plankton bloom in the northern North Sea. II. Dissolved lipids and fatty acids. Mar. Chem. 14: 163-173

Morris, R. J., McCartney, M. J., Robinson, G. A. (1983). Studies of a spring phytoplankton bloom in an enclosed experimental ecosystem. I. Biochemical changes in relation to the nutrient chemistry of water. J. exp. mar. Biol. Ecol, 70: 249-262

Opute, F. I. (1974). Studies on fat accumulation in Nitzschia palea Kutz. Ann. Bot. 38: 889-902

Parrish, C. C. (1986). Dissolved and particulate lipid classes in the aquatic environment. Ph. D. dissertation, Dalhousie University

Parrish, C. C., Ackman, R. G. (1985). Calibration of the Iatroscan-Chromarod system for marine lipid class analyses. Lipids 20: 521-530

Parrish, C. C. (1987). Time series of particulate and dissolved lipid classes during spring phytoplankton blooms in Bedford Basin, a marine inlet. Mar. Ecol. Prog. Ser. 35: 129-139

Richardson, B., Orcutt, D. M., Schwertner, H. A., Martinez, C. L., Wickline, H. E. (1969). Effects of nitrogen limitation on the growth and composition of unicellular algae in continuous culture. Appl. Microbiol. 18: 245-250

Shifrin, N. S., Chisholm, S. (1981). Phytoplankton lipids: interspecific differences and effects of nitrate, silicate and light-dark cycles. J. Phycol. 17: 374-384

Skipnes, O., Eide, I., Jensen, A. (1980). Cage culture turbidostat: a device for rapid determination of algal growth rate. Appl. environ. Microbiol. 40: 318-325

Thomas, W. H., Seibert, D. L. R., Alden, M., Neori, A., Eldridge, P. (1984). Yields, photosynthetic efficiencies and proximate composition of dense marine microalgal cultures. I. Introduction and Phaeodactylum tricornutum experiments. Biomass 5: 181-209

Wangersky, P. J. (1978). Production of dissolved organic matter. In: Kinne, O. (ed.) Marine ecology, Vol. 4, Dynamics. Wiley, Chichester 